\title{
The impact of COVID-19 on trauma patients and orthopedic trauma operations at a single focused training center for trauma in Korea
}

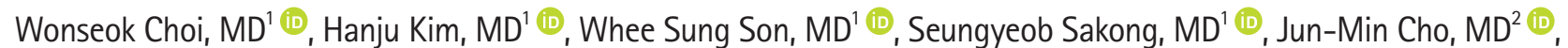

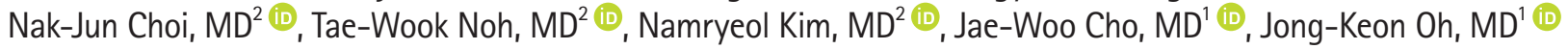 \\ 'Department of Orthopaedic Surgery, Korea University Guro Hospital, Korea University College of Medicine, Seoul, Korea \\ ${ }^{2}$ Department of Trauma Surgery, Korea University Guro Hospital, Korea University College of Medicine, Seoul, Korea
}

Received: September 23, 2021

Revised: November 12, 2021

Accepted: November 20, 2021

Correspondence to

Jong-Keon Oh, MD

Department of Orthopaedic Surgery,

Korea University Guro Hospital, Korea

University College of Medicine, 148

Gurodong-ro, Guro-gu, Seoul 08308,

Korea

Tel: +82-2-2626-3088

E-mail: jkoh@korea.ac.kr
Purpose: The objective of this study was to determine the effects of coronavirus disease 2019 (COVID-19) on the volume of trauma patients, the number of orthopedic trauma operations, and the severity of injuries. We also investigated the correlations between social distancing and these variables.

Methods: This was a retrospective review of trauma patient cases at a single focused training center for trauma in Korea from January 2017 to April 2021. The COVID-19 group included patients treated from January 1 to April 30 in 2020 and 2021, and the control group included patients treated during the same months from 2017 to 2019. The volume of trauma patients according to the level of social distancing was evaluated among patients treated from August 2, 2020 to November 23, 2020.

Results: The study included 3,032 patients who presented to the emergency department with traumatic injuries from January to April 2017 to 2021. The average number of patients was 646.7 and 546.0 in the control and COVID-19 groups, respectively. The percentage of patients injured in traffic accidents (TAs) decreased from $25.0 \%$ to $18.2 \%$ ( $\mathrm{P}<0.0001$ ). The proportions of in-car TAs and pedestrian TAs also decreased from $6.7 \%$ and $10.8 \%$ to $3.5 \%$ and $6.0 \%$, respectively $(\mathrm{P}=0.0002$ and $\mathrm{P}<0.0001)$. The percentage of bicycle TAs increased from $2.4 \%$ to $4.0 \%(\mathrm{P}=0.0128)$. The proportion of patients with an Injury Severity Score above 15 and the mortality rate did not change significantly. As the level of social distancing increased, the number of trauma patients and the number of trauma injuries from TAs decreased. The number of orthopedic trauma operations also depended on the social distancing level.

Conclusions: The number of trauma patients presenting to the emergency department decreased during the COVID-19 period. The volume of trauma patients and orthopedic trauma operations decreased as the social distance level increased.

Keywords: Epidemiology; COVID-19; Trauma; Incidence; Physical distancing 


\section{INTRODUCTION}

Coronavirus disease 2019 (COVID-19) is caused by severe acute respiratory syndrome coronavirus-2, which was first reported in December 2019 in Wuhan, China [1]. The disease spread rapidly around the world and was declared a pandemic by the World Health Organization on March 11, 2020 [2]. The first case in Korea was reported on January 20, 2020 [3]. Currently, almost 270,000 people have been infected in Korea [4]. The spread of the disease has severely altered people's daily lives, as well as the economy and healthcare system.

The incidence of traumatic injuries is influenced by several factors, including the environment and outbreaks of contagious diseases [5-7]. To control the spread of COVID-19, many countries implemented lockdown and/or social distancing policies. In Korea, the government restricted private gatherings according to the number of people with time limitations. In addition, sports facilities and restaurants were closed after designated hours. Social distancing in Korea was divided into three levels and was revised to four levels from July 1, 2020. Level 4 is defined as a pandemic, when the national quarantine system has reached its limit; people are advised to stay home as much as possible, and only private meetings of up to two people are allowed after 6 Pм [4].

The effects of COVID-19 on the volume of trauma patients in several countries have been reported [8-10]. The pandemic has altered the needs and provision of health care throughout the world. As a result of restrictive social measures, such as lockdown or social distancing, the incidence of crimes and traffic accidents (TAs) decreased sharply [11]. The aim of this study was to determine the impact of the pandemic and social distancing policies on the volume of trauma patients at our trauma center. We hypothesized that the COVID-19 pandemic reduced the volume of trauma patients and that taking restrictive actions affected the number of patients who presented to the emergency department due to traumatic injuries.

\section{METHODS}

\section{Ethical statements}

The protocol of the study was reviewed and permission to use data from the Korea Trauma Data Bank (KTDB) and patients' medical charts was obtained from the Institutional Review Board of Korea University Guro Hospital (No. 2021GR0430). The Institutional Review Board waived the requirement for obtaining informed consent because the study was designed to review existing records.

\section{Study design and setting}

An observational retrospective analysis using the KTDB and our hospital's trauma registry was conducted. Our hospital is located in the southwest region of the capital of Korea, with a population of over 9.5 million people. The hospital is a regional emergency center and is designated as a focused training center for trauma with 1,053 beds. It receives around 2,000 trauma patients per year and about 1,500 patients are admitted. In March 2014, our hospital established an urgent treatment processing system to promote quick and effective trauma management. Patients presenting to the emergency department at our institution from January to April in 2017 to 2021 were included in the analysis. The COVID-19 group included patients who arrived at the emergency department from January 1 to April 30 of 2020 and 2021, and the control group included patients who arrived at the emergency department during the same months in 2017 to 2019.

\section{Data collection}

The collected variables included the age and sex of patients, mechanism of injury, time of arrival at the emergency department, Injury Severity Score (ISS), treatment with orthopedic surgery, and mortality. Orthopedic trauma operations were performed by orthopedic trauma surgeons affiliated with our hospital's trauma center. To analyze the effects of social distancing, periods of 14 consecutive days starting from August 2, 2020 to November 23, 2020 were compared. In these periods, the level of social distancing was elevated, lowered, or remained the same. The total number of patients, the number of TA patients, the number of orthopedic trauma operations, the number of patients with an ISS above 15 , and mortality were compared.

\section{Statistical analysis}

The chi-square test was used to compare variables between the COVID-19 group and the control cohort. Poisson regression analysis was used to compare trauma incidence and other variables among different social distancing levels. SAS ver. 9.4 (SAS Institute, Cary, NC, USA) was used to analyze the data. A P-value less than 0.05 was considered to indicate statistical significance.

\section{RESULTS}

The demographic characteristics of the patients are shown in Table 1 . The total number of patients was 3,032, including 1,752 male patients (51.8\%) and 1,280 female patients (42.2\%). The distribution of patients by sex was not statistically different $(\mathrm{P}=0.356)$. The average numbers of patients per year in the con- 
Table 1. Demographic characteristics of patients from January to April, 2017-2021

\begin{tabular}{|c|c|c|c|c|c|c|c|c|}
\hline \multirow{2}{*}{ Characteristic } & \multicolumn{3}{|c|}{ COVID-19 group } & \multicolumn{4}{|c|}{ Control group } & \multirow{2}{*}{ Total } \\
\hline & 2020 & 2021 & Mean & 2017 & 2018 & 2019 & Mean & \\
\hline No. of patients & 622 & 470 & 546.0 & 609 & 636 & 695 & 646.7 & 3,032 \\
\hline Male sex & $359(57.7)$ & $282(60.0)$ & - & $359(58.9)$ & $346(54.4)$ & $406(58.4)$ & - & $1,752(51.8)$ \\
\hline Age (yr) & $56.8 \pm 22.0$ & $57.4 \pm 21.0$ & - & $53.5 \pm 22.7$ & $54.4 \pm 21.8$ & $53.3 \pm 21.7$ & - & $55.0 \pm 22.0$ \\
\hline
\end{tabular}

Values are presented as number, number (\%), or mean \pm standard deviation. COVID-19, coronavirus 2019.

trol and COVID-19 groups were 646.7 and 546.0, respectively. The average number of patients decreased by $15.6 \%$ in the COVID-19 group compared with the number of patients in the control group. The mean age of the patients was $54.9 \pm 21.9$ years.

A comparison of injury mechanisms between the two groups is shown in Table 2. The percentage of patients with TAs decreased from $25.0 \%$ in the control group to $18.2 \%$ in the COVID-19 group $(\mathrm{P}<0.0001)$. The proportions of patients with in-car TAs and pedestrian TAs decreased from $6.7 \%$ to $3.5 \%$ and $10.8 \%$ to $6.0 \%$, respectively $(\mathrm{P}=0.0002$ and $\mathrm{P}<0.0001)$. In contrast, the percentage of patients injured in bicycle TAs increased from $2.4 \%$ in the control group to $4.0 \%$ in the COVID-group $(\mathrm{P}=0.0128)$. The percentage of motorcycle accidents did not differ significantly between the two groups $(5.2 \%$ and $4.8 \%$, respectively, $\mathrm{P}=0.6343$ ). Injuries from slips, falls, or injuries by machines at the workplace did not show statistically significant differences between the two groups.

Table 3 presents a comparison of the percentage of patients with an ISS above 15 and the mortality rate between the two groups. The percentage of patients with an ISS score above 15 (9.6\% and 9.6\% in the control and COVID-19 groups, respectively) and the mortality rate $(2.0 \%$ and $2.6 \%$ in the control and COVID-19 groups, respectively) did not change significantly.

Tables 4, 5 and Fig. 1 show the effects of social distancing on the number of trauma patients, TA, ISS, mortality, and orthopedic trauma operations. Table 6 summarizes the social distancing levels. When the social distancing level increased from self-distancing to level 2, the number of patients presenting to the emergency department per day decreased from 4.71 to 2.93 , and when the social distancing level decreased from level 2 to level 1, the number of patients per day increased from 4.82 to 6.24. Overall, as the level of social distancing increased, the average number of patients decreased, and as the level of social distancing decreased, the number of patients increased. A similar pattern was observed in the number of patients who were injured by TAs, but not at all levels. When the social distancing level decreased from level 2.5 to level 2 , the daily average number of TAs increased from 0.29 to
Table 2. Comparison of trauma patients and mechanisms of injury between the control and COVID-19 groups

\begin{tabular}{lccr}
\hline Injury mechanism & $\begin{array}{c}\text { COVID-19 group } \\
(\mathrm{n}=1,092)\end{array}$ & $\begin{array}{c}\text { Control group } \\
(\mathrm{n}=1,940)\end{array}$ & P-value \\
\hline Traffic accident & $199(18.2)$ & $485(25.0)$ & $<0.0001$ \\
Motorcycle & $52(4.8)$ & $100(5.2)$ & 0.6343 \\
In-car & $38(3.5)$ & $129(6.7)$ & 0.0002 \\
Bicycle & $44(4.0)$ & $47(2.4)$ & 0.0128 \\
Pedestrian & $65(6.0)$ & $209(10.8)$ & $<0.0001$ \\
Slips & $325(29.8)$ & $636(32.8)$ & 0.0861 \\
Falls & $188(17.2)$ & $310(16.0)$ & 0.3776 \\
Machine & $35(3.2)$ & $46(2.4)$ & 0.1716 \\
\hline
\end{tabular}

Values are presented as number (\%).

COVID-19, coronavirus disease 2019.

Table 3. Comparison of the number of patients with an ISS above 15 and mortality

\begin{tabular}{lccc}
\hline Variable & $\begin{array}{c}\text { COVID-19 group } \\
(\mathrm{n}=1,092)\end{array}$ & $\begin{array}{c}\text { Control group } \\
(\mathrm{n}=1,940)\end{array}$ & P-value \\
\hline ISS $>15$ & $105(9.6)$ & $186(9.6)$ & 0.980 \\
Mortality & $28(2.6)$ & $38(2.0)$ & 0.273 \\
\hline
\end{tabular}

Values are presented as number (\%).

ISS, Injury Severity Score; COVID-19, coronavirus disease 2019.

1.21. The number of orthopedic trauma operations changed in the same direction. Neither the number of patients with ISS above 15 nor the mortality rate was affected by social distancing levels. The correlation between the social distancing level and the number of patients was statistically significant in three comparison groups; the number of TA patients was statistically significant in one comparison group, and the number of orthopedic trauma operations was statistically significant in two comparison groups. The number of patients with an ISS $>15$ and the mortality rate did not show significant correlations in any comparison groups.

\section{DISCUSSION}

This study evaluated the impact of the COVID-19 pandemic on 
Table 4. Correlations of social distancing levels with the number of patients, TAs, severity of injury, and orthopedic trauma operations

\begin{tabular}{lccccccc}
\hline Level & \multicolumn{1}{c}{ Date } & $\begin{array}{c}\text { Total } \\
\text { patients }\end{array}$ & Patients/day & TA/day & ISS $>15 /$ day & $\begin{array}{c}\text { Mortality/day } \\
\text { OS operation/ } \\
\text { day }\end{array}$ \\
\hline Self-isolation & $2020.08 .02-2020.08 .15$ & 66 & 4.71 & 0.57 & 0.36 & 0.14 & 1.57 \\
Level 2 & 2020. 08. 16-2020.08.29 & 41 & 2.93 & 0.64 & 0.07 & 0 & 0.79 \\
Level 2.5 & $2020.08 .30-2020.09 .13$ & 28 & 2.21 & 0.29 & 0.14 & 0.07 & 0.21 \\
Level 2 & $2020.09 .14-2020.10 .11$ & 134 & 4.82 & 1.21 & 0.39 & 0.07 & 1.64 \\
Level 1 & 2020.10.12-2020.11.23 & 258 & 6.24 & 1.07 & 0.62 & 0.19 & 1.76 \\
\hline
\end{tabular}

TA, traffic accident; ISS, Injury Severity Score; OS, orthopedic surgery.

Table 5. Poisson regression analysis of differences due to changes in social distancing levels

\begin{tabular}{lccccc}
\hline \multirow{2}{*}{ Difference of level } & \multicolumn{4}{c}{ P-value } \\
\cline { 2 - 6 } & No. of patients & TA & OS operation & No. of patients of ISS $>15$ & Mortality \\
\hline S vs. 2 & 0.016 & 0.808 & 0.056 & 0.103 & 0.157 \\
2 vs. 2.5 & 0.118 & 0.166 & 0.033 & 0.157 & 0.317 \\
2.5 vs. 2 & $<0.001$ & $<0.001^{\text {a) }}$ & $<0.001$ & 0.147 & 0.157 \\
2 vs. 1 & 0.016 & 0.524 & 0.878 & \\
\hline
\end{tabular}

TA, traffic accident; OS, orthopedic surgery; ISS, Injury Severity Score; S, self-isolation.

${ }^{\text {a) }} 0.0002$.

the volume of trauma patients and orthopedic trauma operations at a single trauma center in Korea. A significant decline in trauma patients has been reported worldwide during the COVID-19 period. Our study also showed that the number of patients with traumatic injuries decreased during the COVID-19 era compared to the control group from before the COVID-19 pandemic (Table 1). We postulate that overall movement in our region declined after the outbreak of COVID-19 due to modifications of people's behavior, including decreased time spent at work and social activities. Behavioral change in patients seeking medical assistance may also be a reason for the decline in patients presenting to the emergency department [12]. The COVID-19 pandemic resulted in a redistribution of public health resources; thus, restricted access to the emergency department due to COVID-19 patients may have also been responsible for the overall decline in trauma patients. However, our trauma center remained open during the pandemic period.

The proportion of patients experiencing TAs decreased during the COVID-19 period (Table 2). The percentages of patients injured in in-car TAs and pedestrian TAs decreased, whereas that of patients who experienced bicycle TAs increased significantly. The increased proportion of bicycle accidents may have been caused by the increased use of bicycles rather than public transportation or the use of bicycles for exercising rather than working out in public gyms. Although the number of patients was small, the incidence of motorcycle TAs did not differ between the two groups. Other injury mechanisms did not change significantly.

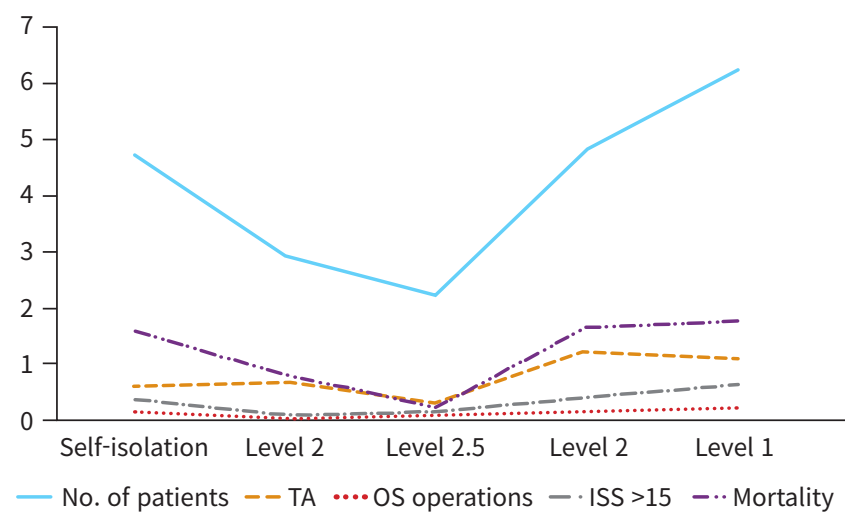

Fig. 1. Patterns of the daily average number of patients, traffic accident (TA) incidents, orthopedic surgery (OS) operations, patients with Injury Severity Score (ISS) $>15$, and mortality depending on social distancing levels.

Some studies have highlighted psychological changes due to quarantine or isolation; an investigation exploring the effects of these psychological changes on self-inflicted injuries would be interesting [13].

Injury severity and mortality were not significantly different between the two patient groups (Table 3). In other words, during the COVID-19 period, patients had injuries comparable to those of patients before the COVID-19 period.

Many countries are taking social actions to slow down or stop the spread of COVID-19. These measures may also prevent accidents. Staying home and limiting social activities may reduce the 
Table 6. Goals and guidelines of social distancing levels

\begin{tabular}{|c|c|}
\hline Social distancing level & Description \\
\hline \multirow[t]{4}{*}{ Self-isolation } & $\begin{array}{l}\text { Goal: allow daily social and economic activities under prevention regulations while managing incidence levels under } \\
\text { the capacity of healthcare system }\end{array}$ \\
\hline & - Social meetings and admissions to sporting events are allowed \\
\hline & - School attendance and online sessions are jointly implemented \\
\hline & - Public institutions operate under the condition of reduced density \\
\hline \multirow[t]{5}{*}{1} & Additional enhanced epidemic control measures are carried out to implement self-isolation level \\
\hline & - Unnecessary visits, meetings, and social events are advised to be avoided \\
\hline & - $1 \mathrm{~m}$ distance between the tables or setting up partitions between the tables at restaurants with no time limits \\
\hline & - One person per $6 \mathrm{~m}^{2}$ area at entertainment facilities \\
\hline & - Limiting the number of audience up to $50 \%$ at sporting events \\
\hline \multirow[t]{4}{*}{2} & Goal: limiting gathering of people to control the spread of the disease in the local region \\
\hline & - Private gatherings with more than nine people are prohibited \\
\hline & - Restaurants and entertainment facilities closed after midnight \\
\hline & - Limiting the number of audience up to $30 \%$ at sporting events \\
\hline \multirow[t]{5}{*}{2.5} & Goal: stop the rapid spread of disease and recover quarantine controls \\
\hline & - All meetings and events with five or more people are prohibited \\
\hline & - Restaurants and entertainment facilities closed after 10 PM \\
\hline & - No school attendance is allowed \\
\hline & - Public institutions are enforced with work-from-home with exceptions \\
\hline
\end{tabular}

number of trauma incidents, including TAs. We evaluated the adjacent social distancing levels to see the effect of changing the social distancing level on the volume of trauma patients. The volume of trauma patients and orthopedic trauma operations decreased when the level of social distancing increased and vice versa (Tables 4, 5). This may have been due to decreased movement and social activities in response to strengthened social distancing. Although not all the results showed statistical significance, the social distancing level was inversely related to the number of trauma patients, TA incidents, and the number of orthopedic trauma operations. These results are in agreement with studies from other countries $[8,10,14,15]$. Neither the number of patients with an ISS $>15$ nor mortality was significantly different in the control group compared with the COVID-19 group. The lack of differences may be explained in two ways. First, the number of cases may have been too small to see the correlation. Second, trauma injuries can be severe regardless of whether the number of patients declines. Even during social distancing, an increase in trauma patients can be anticipated as people become familiar with the restrictive measures.

The COVID-19 pandemic caused major challenges in managing trauma patients $[14,16,17]$. Even in this challenging situation, health care providers and trauma specialists should pay particular attention and be prepared for an increase in patients as social distancing levels decrease. Trauma care specialists should also be aware that severe traumatic injuries can happen at any time and they should be ready to provide support at all times. The protocols that existed before the outbreak of COVID-19 may be difficult to maintain. Protective measures for health care workers might lead to delays in the rapid treatment of critical trauma patients, surgery can be postponed due to protection against COVID-19 or detection of COVID-19 in a patient, and shortages of blood products can lead to insufficient treatment of critical patients. Therefore, many changes are necessary and improvisations based on previous management strategies must be taken $[18,19]$. Appropriate allocation of resources such as space, equipment, and healthcare personnel should take place. Strict use of personal protective equipment should be mandated, along with minimizing the required staff in the trauma bay and operating rooms.

One of the limitations of this study is that it was conducted at a single trauma center located in a specific region. The results of this study do not reflect the situation of all trauma centers, and inconsistent results may be observed across different institutions. To validate the effects of COVID-19 on trauma patients, a nationwide multi-center study with more patients is necessary. A second limitation of this study is that patients were selected in certain periods. To determine the effects of COVID-19, we compared the volume of patients between January and April in 2020 and 2021 to the volume in the corresponding months from 2017 to 2019 . The volume of patients can be affected by weather and 
season, which was the main reason why we excluded patients treated from May to December 2020. To overcome selection bias, we compared the same periods in 3 years before COVID-19. A comparison of patients treated throughout the entire year might provide more accurate insights on the effects of COVID-19 on the volume of trauma patients, but using data from the same period in 2 years for comparison may provide correlations over a longer period. Furthermore, to evaluate the effect of social distancing, the period from August 2, 2020 to November 23, 2020 was analyzed. The level of social distancing remained the same for longer times in other periods. Thus, this period was chosen to determine the effects of changes in social distancing measures on the volume and severity of trauma patients.

In conclusion, the volume of trauma patients and orthopedic trauma operations decreased during the COVID-19 period at our institution. Taking restrictive actions and self-isolation had several trauma-related effects, including a reduction in the number of injuries. A nationwide study is needed for a more conclusive assessment of the correlation between COVID-19 and trauma incidence.

\section{NOTES}

\section{Ethical statements}

The study was obtained from the Institutional Review Board of Korea University Guro Hospital (No. 2021GR0430). The Institutional Review Board waived the requirement for obtaining informed consent because the study was designed to review existing records.

\section{Conflicts of interest}

The author has no conflicts of interest to declare.

\section{Funding}

None.

\section{Author contributions}

Conceptualization: JKO; Data curation: WSS, JMC; Formal analysis: WC; Methodology: HK, TWN; Project administration: JKO, NK; Visualization: SS, NJC; Writing-original draft: WC; Writingreview \& editing: HK, WSS, SS, JMC, NJC, TWN, NK, JWC, JKO. All authors read and approved the final manuscript.

\section{REFERENCES}

1. Zhu N, Zhang D, Wang W, et al. A novel coronavirus from patients with pneumonia in China, 2019. N Engl J Med 2020; 382:727-33.

2. Huang C, Wang Y, Li X, et al. Clinical features of patients infected with 2019 novel coronavirus in Wuhan, China. Lancet 2020;395:497-506.

3. Kim JY, Choe PG, Oh Y, et al. The first case of 2019 novel coronavirus pneumonia imported into Korea from Wuhan, China: implication for infection prevention and control measures. J Korean Med Sci 2020;35:e61.

4. Ministry of Health and Welfare (MOHW). Coronavirus disease-19, Republic of Korea [Internet]. Sejong: MOHW; 2021 [cited 2021 Sep 13]. Available from: http://ncov.mohw.go.kr/.

5. Kim SH, Sul YH, Lee JY, Kim JS. The influence of seasons and weather on the volume of trauma patients: 4 years of experience at a single regional trauma center. J Trauma Inj 2021;34:21-30.

6. Curran T, Bogdanovski DA, Hicks AS, et al. The effects of Hurricane Sandy on trauma center admissions. Eur J Trauma Emerg Surg 2018;44:137-41.

7. Peeri NC, Shrestha N, Rahman MS, et al. The SARS, MERS and novel coronavirus (COVID-19) epidemics, the newest and biggest global health threats: what lessons have we learned. Int J Epidemiol 2020;49:717-26.

8. Pintado JF, Gibaja W, Vallejos RA, Rosas W, Guerra-Farfan E, Nunez JH. How COVID-19 has affected emergent visits to a Latin-American trauma department: experience at a Peruvian national trauma referral center. Injury 2020;51:2834-9.

9. Haffer H, Schomig F, Rickert M, et al. Impact of the COVID-19 pandemic on orthopaedic and trauma surgery in university hospitals in Germany: results of a nationwide survey. J Bone Joint Surg Am 2020;102:e78.

10. DiFazio LT, Curran T, Bilaniuk JW, et al. The impact of the COVID-19 pandemic on hospital admissions for trauma and acute care surgery. Am Surg 2020;86:901-3.

11. Venter A, Lewis CM, Saffy P, Chadinha LP. Locked down: impact of COVID-19 restrictions on trauma presentations to the emergency department. S Afr Med J 2020;111:52-6.

12. Wong JS, Cheung KM. Impact of COVID-19 on orthopaedic and trauma service: an epidemiological study. J Bone Joint Surg Am 2020;102:e80.

13. Papadimos TJ. What's new in critical illness and injury science? Mental health and COVID-19: self-inflicted and interpersonal violence amid a pandemic. Int J Crit Illn Inj Sci 2020;10:45-8.

14. Nunez JH, Sallent A, Lakhani K, et al. Impact of the COVID-19 pandemic on an emergency traumatology ser- 
vice: experience at a tertiary trauma centre in Spain. Injury 2020;51:1414-8.

15. Kamine TH, Rembisz A, Barron RJ, Baldwin C, Kromer M. Decrease in trauma admissions with COVID-19 pandemic. West J Emerg Med 2020;21:819-22.

16. Li Y, Zeng L, Li Z, et al. Emergency trauma care during the outbreak of corona virus disease 2019 (COVID-19) in China. World J Emerg Surg 2020;15:33.

17. Lezak BA, Cole PA Jr, Schroder LK, Cole PA. Global experience of orthopaedic trauma surgeons facing COVID-19: a survey highlighting the global orthopaedic response. Int Orthop 2020;44:1519-29.

18. Dutton RP, Grissom TE, Herbstreit F. COVID-19 and trauma care: improvise, adapt, and overcome! Anesth Analg 2020; 131:323-5.

19. Coleman JR, Burlew CC, Platnick KB, et al. Maintaining trauma care access during the COVID-19 pandemic: an urban, level-1 trauma center's experience. Ann Surg 2020;272:e5860. 